\title{
Relationship of Depression, Anxiety, and Rumination Scores with EEG Connectivity of Resting State Networks
}

\author{
A. V. Bocharov ${ }^{a, b, *}$, G. G. Knyazev ${ }^{a}$, A. N. Savostyanov ${ }^{a, b}$, A. E. Saprygin ${ }^{a}$, \\ E. A. Proshina ${ }^{a}$, and S. S. Tamozhnikov ${ }^{a}$ \\ ${ }^{a}$ Scientific Research Institute of Neuroscience and Medicine, Novosibirsk, 630117 Russia \\ ${ }^{b}$ Novosibirsk State University, Novosibirsk, 630090 Russia \\ *e-mail: bocharov@physiol.ru \\ Received February 26, 2020; revised June 22, 2020; accepted August 20, 2020
}

\begin{abstract}
The aim of the research was to study the effect of depression, anxiety, and rumination scores on the balance of activity of the default mode network and attention networks revealed in the resting state EEG records. Forty-five healthy volunteers ( 24 men aged from 18 to 25 years) participated in the resting state EEG recording. The participants filled in the Beck Depression Inventory-II (BDI II), Ruminative Responses Scale, and Eysenck Personality Profiler. The connectivity measures of resting state networks were calculated in EEG data. The networks were detected by the "seed" method. The effects of depressive symptoms, anxiety, and rumination on the connectivity of the networks were analyzed by the regression method. The depressive symptom scores and the rumination scores were correlated with the dominance of the default mode network over attention networks in the right temporal cortex. The depression scores and the anxiety scores were correlated with the dominance of attention networks over the default mode network in the anterior cingulate cortex. It could be suggested that rumination processes are specific for depressive symptoms and are reflected in the dominance of the default mode network in brain structures associated with the processing of emotional introspection. Common to depressive and anxious symptoms is a state of alertness, which is reflected in the dominance of attention networks in brain structures associated with decision-making.
\end{abstract}

Keywords: EEG, default mode network, attention networks, depression, anxiety, rumination

DOI: $10.1134 / \mathrm{S} 0362119721010023$

An important issue in psychiatry is the differential diagnosis of anxiety and depressive disorders. The symptoms of anxiety and depression are strongly correlated with each other in the population; therefore, some psychiatrists even suggest combining anxiety and depressive disorders into one group of affective disorders [1]. At the level of brain mechanisms, however, these two types of disorders can differ significantly from each other. The discovery of resting state networks provides a new perspective in the study of this issue.

Cognitive processes occurring in the absence of a task requiring conscious processing constitute a significant part of a person's mental life and can determine human behavior in the long term [2]. A number of brain structures are now known to exhibit a high resting metabolic activity and a sustained pattern of deactivation during a wide variety of specific purposeful behaviors. The set of such structures, which includes the medial part of the prefrontal cortex, the posterior cingulate cortex, the precuneus, and the lateral, medial and inferior parts of the parietal cortex, have strong functional interrelationships and form a network that is called the default mode network (DMN)
[3]. At the same time, it has been shown that DMN increases its activity during tasks related to personal memories, when thinking about relationships with other people, in tasks for self-projection, e.g., when perceiving oneself from the point of view of other people or imagining one's future $[2,4]$.

In contrast to $\mathrm{DMN}$, networks involved in the regulation of attention (task-positive networks, TPNs) increase their activity during the transition from a state of rest to the perception of information from the outside [5]. An important indicator of the adequate functioning of the brain is the balance of the activity of DMN and TPNs. Dominance of DMN over TPNs often accompanies pathological conditions and may be associated with impaired attention. This dominance, in particular, was found in patients with major depressive disorder, as well as in clinically healthy subjects with depressive symptoms [6-9]. There are no data on the effect of anxiety or rumination on the ratio of the DMN and TPN activities.

Most research in this area has been done using positron emission tomography (PET) and functional magnetic resonance imaging (fMRI). These methods 
are extremely important and necessary for the precise spatial localization of processes in the brain, but they do not make it possible to study the dynamics of brain processes on a millisecond time scale. It is known that the functioning of the brain is based on the principle of combining the structures of the brain into functional systems necessary for the performance of current functions. According to one point of view, the role of integration into functional systems is performed by oscillations of the bioelectric activity of the brain [10, 11]. Therefore, the study of resting state networks by electrophysiological methods is very important for understanding their role both in the function of a healthy brain and in the occurrence of various kinds of deviations.

The aim of the study was to determine the influence of the severity of depression, anxiety, and rumination on the EEG correlates of the indicators of the connectivity of resting state networks. The objective of the study was to identify the influence of anxiety and depressive symptoms and rumination on the balance of the activity of DMN and TPNs, the connectivity indicators of which were assessed at rest on the basis of EEG data. Since an increased anxiety is considered a risk factor for depression and accompanies depressive states [12], but, on the other hand, depression is not limited to anxiety, we expected to find both elements of similarity and differences in the influence of these two factors. It is also known that subjects with depressive symptoms are characterized by the dominance of negative thoughts, as well as the difficulty of getting rid of them [13]. We expected to find similarities in the influence of the severity of symptoms of depression and the severity of rumination on the balance of the DMN and TPN activities.

\section{METHODS}

The study involved 45 healthy right-handed volunteers, including 24 men (aged 18 to 25 years, mean $=$ 20.8 years, standard deviation $=2.6$ years). The study participants were right-handed with normal vision or vision corrected to normal, healthy, and drug-free.

Before the study, the participants filled out a questionnaire in which they noted whether they have mental or physical disabilities or health problems, assessed their current state of health, and also answered questions about the use of psychoactive substances. Women answered questions about the phase of the menstrual cycle and the use of hormonal contraceptives.

EEG recording and experimental paradigm. EEG recording was performed using a cap with 128 electrodes. The coordinates of the electrodes of each participant in the study were determined using a digitizer (Polhemus, United States). During the EEG recording, the study participants sat in a dimly lit and soundproofed room. The resting EEG was recorded for
12 min (6 min with the eyes closed and 6 min with the eyes open). After recording, the subjects filled out questionnaires: Beck's questionnaire (Beck) on the severity of depressive symptoms [14], Eysenck's personality questionnaire (Eysenck) [15], and a questionnaire on the severity of rumination [16].

Analysis of EEG data. Artifacts were removed using the analysis of independent components in the EEGlab software. After removing artifacts, the EEG data was filtered in five standard frequency ranges. After filtration, cortical sources of electrical activity were localized by spatial filtration. Correlation of electrode positions with the MNI standard was performed using the SPM-12 software based on the measured coordinates of electrodes and reference points on the surface of the head (nasion, preauricular points). This allowed subsequent group statistical analysis. The next step was the correction of the "signal leakage" arising from the poor spatial resolution of the source localization method. For this, the orthogonalization method was used [17, 18]. After orthogonalization, the Gilbert transform was applied and the signal envelope was calculated, which, after reducing the digitization frequency to $1 \mathrm{~s}$, was used to construct connectivity maps [19]. We used the "seed" method to build connectivity maps. It is important to identify three networks that are most directly related to the topic of our research. This is, first of all, DMN, which is most active at rest and is associated with psychological processes of selfawareness. In addition, these are the networks involved in the regulation of attention and working memory processes: the central executive network (CEN, central executive network) and the salience network (SN). We used the "seed" method to identify resting state networks used earlier [8, 9]. For DMN, the medial prefrontal cortex, the posterior cingulate gyrus, and the left and right parietal cortices were chosen as the "seed" [20]. For the CEN, the left and right dorsolateral cortices were used as a "seed"; and for the $\mathrm{SN}$, the left and right insular cortices [5]. Connectivity maps were calculated for each subject and then used for statistical analysis in the SPM-12 software package.

\section{RESULTS}

To analyze the effect of depressive symptoms, anxiety and rumination on the connectivity indicators of DMN and TPN, a multivariate design was used in the SPM-12 software package. The relationship between psychometric variables and connectivity indicators was revealed by the regression method. The control of false positive effects was carried out using a double confidence threshold at the voxel level $(p<0.001)$ and at the cluster level (adjusted $p<0.001$ ). To reveal the effect of psychometric variables on the ratio of connectivity between DMN and TPN, their regression was performed on the contrast DMN $>$ TPN, or DMN $<$ TPN. The DMN $>$ TPN contrast reveals the 

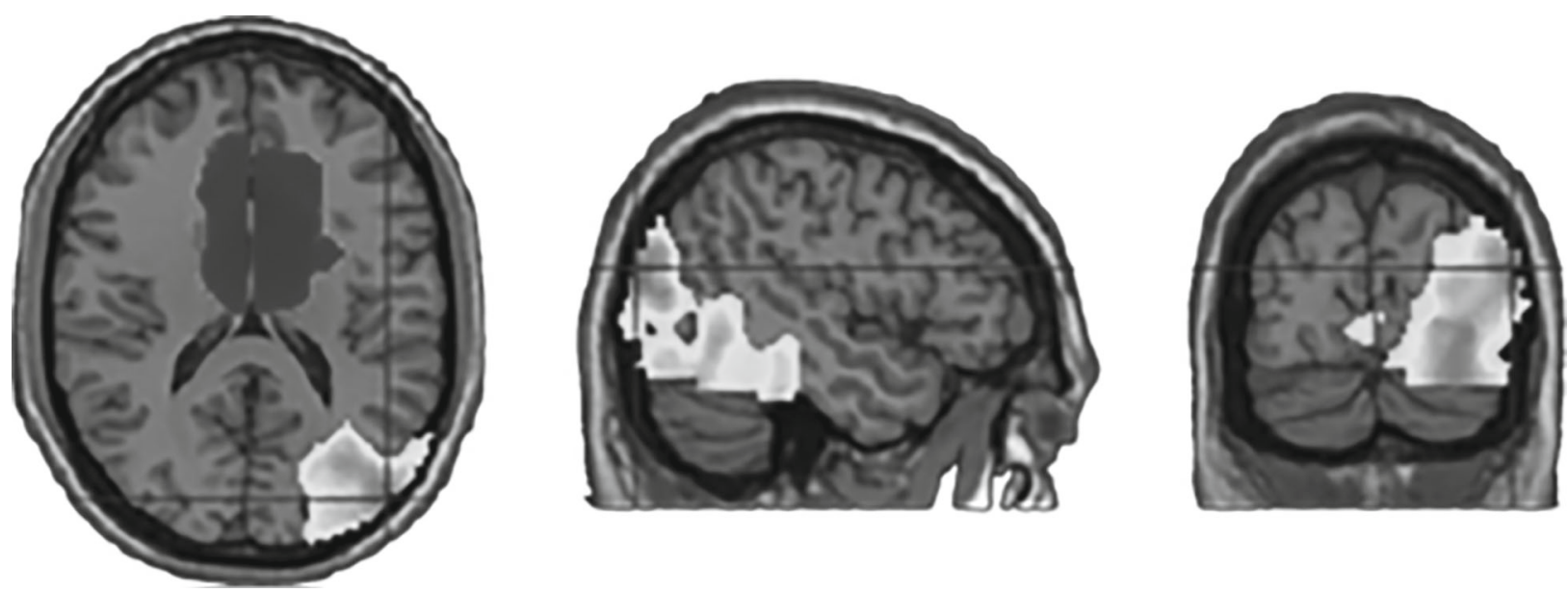

Fig. 1. Regression of assessments of depressive symptoms for the DMN $>$ TPN contrast (light tone) and for the DMN $<$ TPN contrast (dark tone).
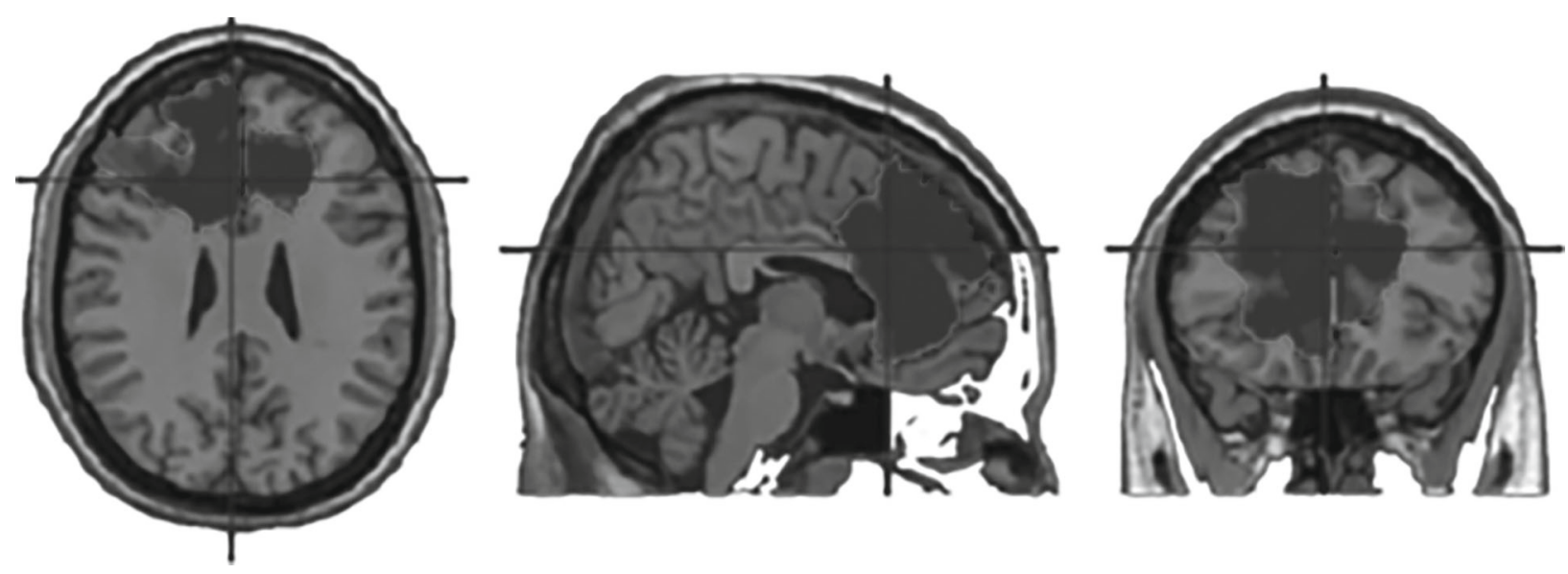

Fig. 2. Regression of assessments of anxiety for the DMN $<$ TPN contrast (dark tone).

effects of DMN dominance over TPN, and the DMN $<$ TPN contrast reveals the effects of TPN dominance over DMN.

Regression of estimates of depressive symptoms on the DMN > TPN contrast revealed a significant effect in the right temporal region, including the amygdala, insula, and parahippocampal gyrus. Regression of the assessments of depressive symptoms for the DMN < TPN contrast revealed a significant cluster in the anterior cingulate gyrus (Fig. 1). In Fig. 1, the relationship between depression and the dominance of DMN is shown in light colors, and that with the dominance of TPN, in dark colors.

Regression of assessments of anxiety on the DMN > TPN contrast did not reveal significant effects. Regression of anxiety assessments for the DMN < TPN contrast revealed a significant cluster centered in the anterior cingulate gyrus (Fig. 2).
Regression of rumination estimates for the DMN > TPN contrast revealed a significant cluster in the right parietal area (Fig. 3). Regression of the rumination scale scores on the DMN $<$ TPN contrast did not reveal significant effects.

Under the control of the rumination scale, regression of the assessments of depressive symptoms on the DMN > TPN contrast did not reveal significant effects.

\section{DISCUSSION}

According to the data obtained, the severity of depressive symptoms was correlated with the dominance of DMN in the right temporal cortex. The significant cluster included not only the cortical regions of the temporal lobe, but also deeper areas of the gray matter, including the amygdala. All of these structures are involved in a wide range of emotional processes. It can be assumed that the dominance of DMN over 

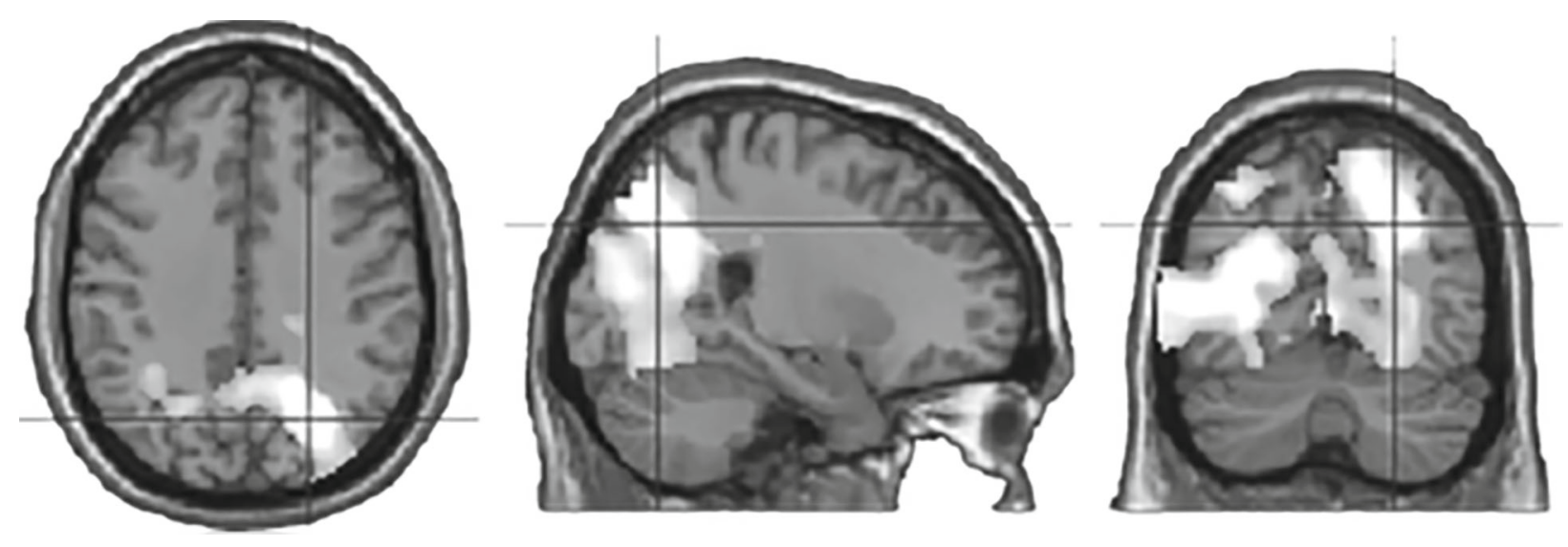

Fig. 3. Regression of assessments of rumination for the DMN $>$ TPN contrast (light tone).

TPN in this area of the brain indicates that the emotional sphere is under stronger control of introspective processes and reacts less to signals coming from outside. The study tested the effect of rumination on the balance of activity of resting state networks, which has been found to be similar to the effect of depressive symptoms in the dominance of DMN in the right temporal cortex. It can be assumed that processes of emotional introspection are specific for depressive symptoms and rumination [21], which are reflected in the dominance of the DMN in the brain structures associated with emotional processing. The results obtained correspond to the well-known fact about the predominance of the processes of emotional rumination in depression and about the reduced sensitivity to signals from the outside world [6,7].

At the same time, depressive symptoms in our sample were correlated with the dominance of the TPN over the DMN in the anterior cingulate gyrus. This area of the cortex is involved in a wide range of decision-making and behavior control processes, including the control of emotional responses. Interestingly, only the latter effect was found for anxiety. The dominance of the TPN in this area of the brain may indicate a tendency to make decisions based on external circumstances. This may be due to the state of alertness characteristic of anxious individuals. Thus, one can think that the state of alertness is common for depression and anxiety, which is reflected in the dominance of the TPN in the field of decision-making, and the processes of emotional introspection, which are reflected in the dominance of the DMN in the processing of emotional information, are specific for depressive symptoms and rumination. In interpreting these data, it should be borne in mind that our sample consisted of healthy subjects in whom depressive symptoms did not reach the clinical level. It can be assumed that, at the stage of clinical depression, the manifestations associated with anxiety may play a lesser role, and the process of emotional rumination becomes dominant.

\section{CONCLUSIONS}

Thus, the state of alertness is common to depression and anxiety, which is reflected in the dominance of the TPNs in decision-making. The processes of emotional introspection, which are reflected in the dominance of the DMN in the processing of emotional information, are specific for depressive symptoms and rumination.

\section{ACKNOWLEDGMENTS}

We are grateful to Nadezhda V. Dmitrienko for her help with the study.

\section{FUNDING}

The study was supported by the federal budget for basic scientific research (research no. AAAA-A16116021010228-0, data collection and analysis) and RFBR (project No. 18-00-00939, development of data analysis methods, project No. 20-013-00404, preparation of the article, project No. 18-415-140021, additional data collection).

\section{COMPLIANCE WITH ETHICAL STANDARDS}

All studies were carried out in accordance with the principles of biomedical ethics, formed in the 1964 Declaration of Helsinki and its subsequent updates, and approved by the local bioethical committee of the Scientific Research Institute of Neuroscience and Medicine (Novosibirsk).

\section{INFORMED CONSENT}

Each study participant provided a voluntary written consent signed by him after explaining to him the potential risks and benefits, as well as the nature of the upcoming study.

HUMAN PHYSIOLOGY Vol. $47 \quad$ No. 2 2021 


\section{CONFLICT OF INTEREST}

The authors declare no obvious and potential conflicts of interest related to the publication of this article.

\section{OPEN ACCESS}

This article is licensed under a Creative Commons Attribution 4.0 International License, which permits use, sharing, adaptation, distribution and reproduction in any medium or format, as long as you give appropriate credit to the original author(s) and the source, provide a link to the Creative Commons licence, and indicate if changes were made. The images or other third party material in this article are included in the article's Creative Commons licence, unless indicated otherwise in a credit line to the material. If material is not included in the article's Creative Commons licence and your intended use is not permitted by statutory regulation or exceeds the permitted use, you will need to obtain permission directly from the copyright holder. To view a copy of this licence, visit http://creativecommons.org/licenses/by/4.0/.

\section{REFERENCES}

1. De Bolle, M. and De Fruyt, F., The tripartite model in childhood and adolescence: Future directions for developmental research, Child Dev. Perspect., 2010, vol. 4, no. 3, p. 174.

2. Mason, M.F., Norton, M.I., van Horn, J.D., et al., Wandering minds: the default network and stimulusindependent thought, Science, 2007, vol. 315, no. 5810, p. 393.

3. Raichle, M.E., MacLeod, A.M., Snyder, A.Z., et al., A default mode of brain function, Proc. Natl. Acad. Sci. U.S.A., 2001, vol. 98, no. 2, p. 676.

4. Raichle, M.E., The brain's default mode network, $A n$ nu. Rev. Neurosci., 2015, vol. 38, p. 433.

5. Seeley, W.W., Menon, V., Schatzberg, A.F., et al., Dissociable intrinsic connectivity networks for salience processing and executive control, J. Neurosci., 2007, vol. 27 , no. 9, p. 2349.

6. Hamilton, J.P., Furman, D.J., Chang, C., et al., Default-mode and task-positive network activity in major depressive disorder: implications for adaptive and maladaptive rumination, Biol. Psychiatry, 2011, vol. 70, no. 4 , p. 327.

7. Hamilton, J.P., Chen, M.C., and Gotlib, I.H., Neural systems approaches to understanding major depressive disorder: an intrinsic functional organization perspective, Neurobiol. Dis., 2013, vol. 52, p. 4.

8. Knyazev, G.G., Savostyanov, A.N., Bocharov, A.V., et al., Task-positive and task-negative networks and their relation to depression: EEG beamformer analysis, Behav. Brain Res., 2016, vol. 306, p. 160.

9. Knyazev, G.G., Savostyanov, A.N., Bocharov, A.V., et al., Task-positive and task-negative networks in major depressive disorder: a combined fMRI and EEG study, J. Affect. Dis., 2018, vol. 235, p. 211.

10. Buzsáki, G. and Draguhn, A., Neuronal oscillations in cortical networks, Science, 2004, vol. 304, no. 5679, p. 1926.

11. Nunez, P.L., Toward a quantitative description of large-scale neocortical dynamic function and EEG, Behav. Brain Sci., 2000, vol. 23, no. 3, p. 371.

12. McElroy, E., Fearon, P., Belsky, J., et al., Networks of depression and anxiety symptoms across development, J. Am. Acad. Child Adolesc. Psychiatry, 2018, vol. 57, no. 12, p. 964.

13. Joormann, J., Yoon, K.L., and Zetsche, U., Cognitive inhibition in depression, Appl. Prev. Psychol., 2007, vol. 12, no. 3, p. 128.

14. Beck, A.T., Steer, R.A., and Brown, G.K., Manual for the Beck Depression Inventory-II, San Antonio, TX: Psychological Corp., 1996.

15. Eysenck, H.J. and Wilson, G.D., Manual of the Eysenck Personality Profiler, Guildford: Psi-Press, 2000.

16. Treynor, W., Gonzalez, R., and Nolenó-Hoeksema, S., Rumination reconsidered: a psychometric analysis, Cognit. Ther. Res., 2003, vol. 27, no. 3, p. 247.

17. Brookes, M.J., Liddle, E.B., Hale, J.R., et al., Task induced modulation of neural oscillations in electrophysiological brain networks, NeuroImage, 2012, vol. 63, no. 4, p. 1918.

18. Hipp, J.F., Hawellek, D.J., Corbetta, M., et al., Largescale cortical correlation structure of spontaneous oscillatory activity, Nat. Neurosci., 2012, vol. 15, no. 6, p. 884 .

19. Brookes, M.J., Woolric, M., Luckhoo, H., et al., Investigating the electrophysiological basis of resting state networks using magnetoencephalography, Proc. Natl. Acad. Sci. U.S.A., 2011, vol. 108, no. 40, p. 16783.

20. Gusnard, D.A. and Raichle, M.E., Searching for a baseline: functional imaging and the resting human brain, Nat. Rev. Neurosci., 2001, vol. 2, no. 10, p. 685.

21. Nolen-Hoeksema, S., Wisco, B.E., and Lyubomirsky, S., Rethinking rumination, Perspect. Psychol. Sci., 2008, vol. 3 , no. 5, p. 400. 